
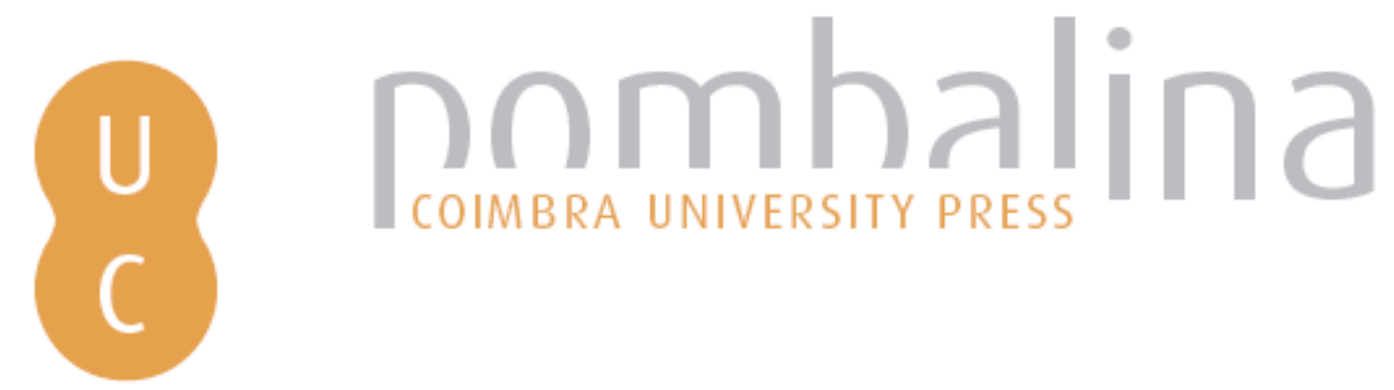

\title{
A história das ciências em Coimbra e o diálogo interdisciplinar: os professores Joaquim de Carvalho, Luís de Albuquerque e Rómulo de Carvalho
}

Autor(es): $\quad$ Fiolhais, Carlos

Publicado por: Imprensa da Universidade de Coimbra

URL

persistente: URI:http://hdl.handle.net/10316.2/40064

DOI: $\quad$ DOI:https://doi.org/10.14195/978-989-26-1239-3_3

Accessed : $\quad$ 26-Apr-2023 12:05:01

A navegação consulta e descarregamento dos títulos inseridos nas Bibliotecas Digitais UC Digitalis, UC Pombalina e UC Impactum, pressupõem a aceitação plena e sem reservas dos Termos e Condições de Uso destas Bibliotecas Digitais, disponíveis em https://digitalis.uc.pt/pt-pt/termos.

Conforme exposto nos referidos Termos e Condições de Uso, o descarregamento de títulos de acesso restrito requer uma licença válida de autorização devendo o utilizador aceder ao(s) documento(s) a partir de um endereço de IP da instituição detentora da supramencionada licença.

Ao utilizador é apenas permitido o descarregamento para uso pessoal, pelo que o emprego do(s) título(s) descarregado(s) para outro fim, designadamente comercial, carece de autorização do respetivo autor ou editor da obra.

Na medida em que todas as obras da UC Digitalis se encontram protegidas pelo Código do Direito de Autor e Direitos Conexos e demais legislação aplicável, toda a cópia, parcial ou total, deste documento, nos casos em que é legalmente admitida, deverá conter ou fazer-se acompanhar por este aviso.

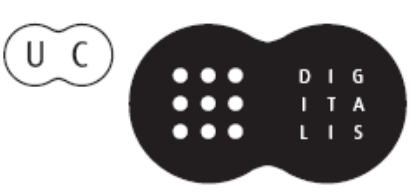




\section{Conrefencicas

INTERDISCIPLINARIDADE E UNIVERSIDADE

ANTÓNIO RAFAELAMARO ÁLVARO GARRIDO JOÃO PAULO AVELÃS NUNES

COORDENAÇÃO 


\title{
A HISTÓRIA DAS CIÊNCIAS EM COIMBRA
} E O DIÁLOGO INTERDISCIPLINAR:

\author{
Os Professores Joaquim De Carvalho, \\ Luís De Albuquerque E Rómulo De Carvalho
}

Carlos Fiolhais Universidade de Coimbra

\section{RESUMO}

A história da Ciência na Universidade de Coimbra liga-se à história da Ciência em Portugal. Partindo do debate essencial sobre a unidade das ciências, suscitado no III Congresso Internacional de História da Ciência que teve lugar em Portugal em 1934, este texto invoca três personalidades eclécticas que deram um alto contributo para a valorização da história da Ciência. Apresentam-se casos exemplares que mostram a procura da unidade das ciências na Universidade de Coimbra no século XX: Joaquim de Carvalho, Luís de Albuquerque e Rómulo de Carvalho. Com formações distintas, todos eles pugnaram pela difusão da ciência sob a forma privilegiada do livro e todos publicaram extensas bibliografias. A forma como praticaram o cruzamento das ciências com as letras recorrendo à história da ciência nas suas épocas fundamentais (Renascimento e Iluminismo) podem inspirar projectos de interdisciplinaridade na Universidade de Coimbra e apelar à convergência das ciências em geral. 
PAlAVRAS-CHAVE: Ciência; Ciências; Interdisciplinaridade; História; Universidade de Coimbra

\section{ABSTRACT}

The bistory of Sciences at The University of Coimbra and the interdisciplinary dialogue: Joaquim de Carvalbo, Luís de Albuquerque and Rómulo de Carvalho.

The history of Science at the Coimbra University is connected to the history of science in Portugal as a whole. Departing from the essential debate on the sciences unity, pointed out at the Third International Conference of Science History that took place in Portugal in 1934, this text invokes three eclectic personalities who gave a high contribute to the sciences history valorisation. We present three significant cases having in common the search for Science's unity at the Coimbra University in the 20th century: Joaquim de Carvalho, Luís de Albuquerque and Rómulo de Carvalho. With different scientific backgrounds, all of them diffused science book's editions and published extended bibliographies. The way they crossed exact and Natural Sciences with Humanities using science's history in its fundamental ages (Renaissance and Enlightenment) may inspire interdisciplinary projects at Coimbra University and call to science's unity in general.

KEYWORDS: Science; Sciences; Interdisciplinarity; History; University of Coimbra

De 30 de Setembro a 6 de Outubro de 1934, sob o alto patrocínio do Presidente da República Portuguesa General Óscar Carmona e com um comité de honra que incluía o Presidente do Conselho de Ministros Oliveira Salazar, realizou-se em Portugal o III Congresso Internacional de História da Ciência. Foi uma rara ocasião pois um dos maiores eventos da história da ciência mundial tinha lugar entre nós, numa altura em que o mundo se aproximava, talvez sem 
plena consciência, da Segunda Guerra Mundial. e o país vivia as dificuldades e vicissitudes do início do Estado Novo.

O primeiro Congresso tinha sido realizado em Paris em 1929. O segundo em Londres em 1931. E o terceiro estava previsto para Berlim em 1934. Dificuldades da sua realização na capital alemã, associadas à chegada ao poder do nacional-socialismo, originaram uma proposta da sua realização na Península Ibérica, numa organização conjunta de Espanha e Portugal, sendo o tema central - a herança científica árabe - apropriado ao sítio. No entanto, problemas com o grupo espanhol de História das Ciências (recorde-se que a Guerra Civil irromperia em Espanha em 1936) fizeram com que o Congresso tivesse lugar apenas em Portugal.

As sessões realizaram-se no Porto, em Coimbra e em Lisboa. A Universidade de Coimbra desempenhou um papel importante desde o início da organização, pois o Professor da Faculdade de Letras Joaquim de Carvalho, Presidente da Secção de Coimbra do Grupo Português de História das Ciências, tinha-se em 1933 oferecido para organizar as sessões na cidade universitária, assim como algumas excursões na região. Os seus colegas de Coimbra Diogo Pacheco de Amorim (matemático), Geraldino Brites (médico) e Alberto Cupertino Pessoa (também médico, secretário geral do comité organizador) colaboraram com ele e com professores do Porto e Lisboa na preparação da iniciativa. A 2 de Outubro os congressistas visitaram a Biblioteca Joanina, onde lhes foi proporcionada uma exposição de livros de Medicina dos séculos XV e XVI, e onde foram recebidos pelo Reitor. Na Sala Gomes Teixeira realizou-se no mesmo dia a sessão trienal da Academia Internacional de História das Ciências, presidida pelo Doutor George Sartor, o químico de origem belga e radicado nos Estados Unidos que é geralmente visto como o fundador da História da Ciência e que foi fundador e durante muitos anos editor-chefe da revista Isis, uma referência nesta área (pude adquirir num alfarrabista um livro oferecido por George Sarton a Alberto Pessoa, com 
dedicatória em que ele agradecia a hospitalidade em Portugal ${ }^{1}$ ). No dia 3 tiveram lugar sessões de trabalho sobre a história da Medicina (com o foco na medicina árabe). E realizou-se uma sessão na Sala dos Capelos sobre $A$ medicina e os médicos na expansão mundial dos Portugueses, a cargo do Professor de Medicina da Universidade de Lisboa Ricardo Jorge. Finalmente, no dia 4 houve uma outra sessão plenária, da responsabilidade do Professor Comandante Fontoura da Costa, sobre A ciência náutica dos portugueses na época dos Descobrimentos. As excursões incluíram, para além de visita aos principais monumentos da cidade, uma visita ao Buçaco.

Em jeito de conclusão, o secretário da secção de Lisboa do grupo português de História das Ciências Arlindo Camilo Monteiro (um médico interessado pela história da arte) escreveu no Prefácio das Actas, publicadas em 1936, nas quais o retrato do Presidente Carmona, em traje de gala, surge logo a abrir ${ }^{2}$ :

Evocando a Coimbra dos velhos tempos, com tanto mais entusiasmo quanto a contemporânea nos parecia em grande parte ausente ou, pelo menos, em férias, o nosso pensamento dirigia-se para os poetas, oradores, escritores e filósofos, entre os quais aqueles que fizeram florir uma filosofia perpetuada sob a designação de Filosofia Conimbricensis, assim como aos espiritos particularmente devotados às ciências experimentais e às artes liberais. Diante da Minerva portuguesa, sonhámos que seria desejável que ela pudesse animar as gerações futuras na tarefa, sem dúvida muito complexa, mas não menos meritória, de estabelecer, com sinceridade e sob todos os pontos de vista, o balanço da sua influência espiritual e social no curso dos tempos.

\footnotetext{
${ }^{1}$ Sarton, 1931. Dedicatória: To Professor Alberto Pessoa in remembrance of the happy days of Coimbra and Lisboa.

2 Vários, 1936.
} 
O Professor Joaquim de Carvalho foi, portanto, um dos principais animadores do Congresso. Nele apresentou uma comunicação, que consta das referidas Actas, sobre Jacob de Castro Sarmento e a introdução das concepções de Newton em Portugal. Recorde-se que Castro Sarmento propôs, embora sem a devida concretização, a tradução de latim para português das obras do filósofo Francis Bacon em Portugal e foi o primeiro a publicar uma tradução comentada de um texto do físico Isaac Newton (tinham passado dez anos após a morte de Newton).

Começando por invocar a figura de Joaquim de Carvalho pretendemos aqui falar da necessidade de interdisciplinaridade, da ligação entre as disciplinas, referindo em particular o caso da História das Ciências e alguns dos seus cultivadores em Coimbra. A História das Ciências é um lugar onde o diálogo e a convergência entre as ciências e as letras, áreas tradicionalmente separadas (na Universidade de Coimbra cultivadas em Faculdades próximas fisicamente, mas demasiadas vezes afastadas academicamente), são particularmente propícios. A história é um ramo das Ciências Sociais. A história das ciências ditas "duras", como as tradicionalmente chamadas Ciências Exactas (Matemática, Física e Química) e as Ciências Naturais (Biologia e Geologia), ou mesmo a das ciências menos "duras", como as Ciências da Saúde (Medicina e Farmácia), exige o diálogo interdisciplinar entre historiadores e cientistas das referidas disciplinas. Se, por vezes, é o historiador que se torna historiador de ciência nas outras vezes é o cientista que se torna historiador pelo gosto do conhecimento do passado da sua disciplina, que de resto lhe oferece um melhor conhecimento da sua ciência e, portanto, uma melhor preparação para o seu cultivo. A ciência é acumulação e, para se perceber melhor o método da ciência, não há como olhar para o trabalho de acumulação de conceitos, teorias e experiências, devidamente peneirados pela crítica, que foi efectuado no passado.

Esse diálogo nem sempre é fácil pois, apesar da muito justificável designação unificadora de "ciências", as Ciências Sociais, as Ciências 
Exactas, as Ciências Naturais e as Ciências da Saúde perseguem objectivos distintos e têm metodologias próprias. Divergem também na especificidade da linguagem que utilizam, que, no caso das ciências mais "duras", pode ser bastante hermética para quem não possuir o domínio da Matemática. Ora, para se conhecer a ciência do passado (que deve ser vista, ou melhor revista, mais com os olhos do passado do que com os olhos do presente), tem necessariamente de se conhecer os objectivos, as metodologias e as linguagens das ciências. Em suma e não dizendo nada de novo: para a História da Ciência o concurso dos especialistas de cada disciplina é imprescindível.

Apresentamos aqui três casos exemplares (são, diga-se desde já, casos raros) que mostram a busca da unidade das ciências no século XX na Universidade de Coimbra: as obras do já referido Joaquim de Carvalho (1892-1958), professor de Filosofia que se interessou pela História da Ciência em Portugal, Luís de Albuquerque (1917-1992), professor de Matemática que se interessou pela História da Ciência em Portugal, muito em particular a História da Náutica, e Rómulo de Carvalho (1906-1996), que, sem ter sido professor universitário, foi um dos maiores polímatos lusos do século transacto, com uma obra extraordinária no ensino da Física e da Química, na História da Ciência em Portugal, em particular do século XVIII, e na Poesia.

\section{Joaquim de Carvalho}

Nascido na Figueira da Foz, Joaquim de Carvalho licenciou-se em Direito e Letras, tendo adquirido formação específica na área da Filosofia, embora estivesse próximo da História da Ciência (tinha publicado a sua tese de doutoramento em Coimbra em 1916 sobre António de Gouveia e o Aristotelismo). No quadro da História da Ciência, focou em particular três dos maiores nomes da ciência 
nacional: Pedro Nunes, o já referido Jacob de Castro Sarmento e João Jacinto Magalhães, o primeiro matemático, expoente do quinhentismo português e os outros dois, um médico e o outro instrumentista, insignes figuras do século das Luzes. Carvalho percebeu a relevância desses dois períodos da História da Ciência em Portugal e contribuiu para a sua ênfase: por um lado, Pedro Nunes, que foi contemporâneo de outras grandes figuras da ciência mundial como os médicos Garcia da Orta, Amato Lusitano e Francisco Sanches e o geofísico (para adoptar a nomenclatura de hoje) e homem de Estado D. João de Castro, e, por outro lado, dois "estrangeirados", que contribuíram à distância para o progresso nacional no Iluminismo. $\mathrm{O}$ texto sobre Sarmento encontra-se republicado no volume $\mathrm{V}$ das suas Obras Completas ${ }^{3}$, saídas do prelo da Fundação Calouste Gulbenkian, juntamente com outros estudos de História da Ciência, a maior parte dos quais sobre Pedro Nunes (Joaquim de Carvalho foi o principal impulsionador da publicação, que não chegou ao seu termo devido ao seu falecimento, da Obra de Pedro Nunes, a cargo da Academia das Ciências de Lisboa ${ }^{4}$ ) e a sua obra, mas também sobre Castro Sarmento e Jacinto Magalhães. Nesse mesmo volume $\mathrm{V}$, mostrando a sua enorme polivalência e erudição, estão reunidos os seus estudos de História e Crítica Literária que abrangem vários autores, de Frei Heitor Pinto a Teixeira de Pascoaes, passando por Uriel da Costa, Antero de Quental e os seus colegas na Faculdade de Letras de Coimbra Carolina Michaëlis de Vasconcelos e Eugénio de Castro. Carvalho ficou, além do mais, conhecido como director da Imprensa da Universidade de Coimbra, lugar que ocupou a partir de 1921 e que só foi interrompido pelo governo de António de Oliveira Salazar em 30 de junho de 1934, precisamente o ano do

\footnotetext{
3 Carvalho, 1987.

4 Obras / Pedro Nunes. - Nova edição, revista e anotada por uma Comissão de Sócios da Academia das Ciências. Lisboa: Imprensa Nacional, 1940-1960, 4 vols.
} 
Congresso, por ver naquela editora uma fonte de contestação política. Algumas das suas obras, publicadas pela Fundação Gulbenkian em forma impressa, têm acesso online ${ }^{5}$.

A sua posição sobre a ampla visão que ele entendia necessária ao espírito humano talvez ressalte desta frase que proferiu em 1932 no final de um discurso na Academia de Ciências de Lisboa, quando foi inaugurado Instituto de Altos Estudos (a Academia de Lisboa tinha convidado o professor de Coimbra para ocupar a cátedra de História da Ciência) ${ }^{6}$ :

O homem que apenas explica cientificamente é uma determinação limitada da natureza humana, assim como o é o homem que apenas se move no reino dos valores estéticos, éticos ou religiosos. o grande problema para nós, hoje, é um problema de integração e não de exclusão, e portanto o homem ideal será aquele que substitua a visão unilateral pela visão integral, e se situe numa atitude de compreensão e de vida tal, que realizando um outro tipo humano, demandando com igual intensidade e fervor o conhecimento que explica e o conhecimento que salva, a ambos afinal contenha e supere.

O humanista e historiador do humanismo português José de Pina Martins, membro da Academia das Ciências e grande bibliófilo, diz no seu prefácio a essa obra ${ }^{7}$ :

Só quem não conhecesse Joaquim de Carvalho poderia admirar-se da mestria com que tratava com igual competência problemas de filosofia, de crítica literária, de história da ciência e mesmo de bibliofilia.

\footnotetext{
5 http://joaquimdecarvalho.org/ (acesso em 8 de Setembro de 2014).

6 Carvalho, 1987.

7 Carvalho, 1987
} 


\section{Luís Albuquerque}

O Professor Luís de Albuquerque foi outro extraordinário homem de cultura que marcou o nosso século $\mathrm{Xx}$, juntando de forma rara as ciências e das letras. Natural de Lisboa, estudou Matemática e Engenharia Geográfica na Universidade de Coimbra, tendo-se doutorado em 1959 com uma tese intitulada Sobre a Teoria da Aproximação Funcional. Só mais tarde se interessou pela História das Ciências e das Técnicas, muito em particular pela História da Náutica, na qual tão importante foi o contributo dos navegadores portugueses dos séculos XV e XVI. Colaborou com o historiador Armando Cortesão (de origem engenheiro agrónomo) em Coimbra num gabinete de estudos e em cursos sobre cartografia antiga. A sua excelente bagagem matemática permitiu-lhe lidar com grande à vontade com questões complexas, de natureza científica, que colocariam sérias dificuldades a historiadores de formação tradicional. Luís Albuquerque, professor catedrático na Faculdade de Ciências da Universidade de Coimbra desde 1966, foi um autodidacta em história: chegou a um lugar cimeiro nessa área graças à sua enorme capacidade intelectual e a décadas de estudos em História. Não admira por isso que a Universidade de Lisboa, em cuja Faculdade de Letras ensinou, lhe tenha atribuído em 1985 o grau de Doutor honoris causa em História.

Luís de Albuquerque não só desenvolveu a subdisciplina da História da Ciência e da Tecnologia, na senda de outros historiadores da náutica como o já referido comandante Fontoura da Costa, como procedeu a um notável trabalho de divulgação da gesta marítima portuguesa para o grande público. Refiram-se, em particular, os livros Os Descobrimentos Portugueses ${ }^{8}$, dois com o mesmo título destinados a públicos díspares, e Navegadores, Viajantes e

\footnotetext{
8 Albuquerque, 1985, Albuquerque, 1991-1992.
} 
Aventureiros Portugueses. Sécs. $X V$ - $X V I^{9}$, para além da edição de obras de referência como a Biblioteca da Expansão Portugueses ${ }^{10}$, Portugal no Mundo ${ }^{11}$ e Dicionário de História dos Descobrimentos Portugueses $^{12}$. Apesar dessa muito bem sucedida deriva para a História, que lhe passou a ocupar a maior parte do tempo, nunca perdeu a ligação às suas origens. Assim, contribuiu para a formação de um grupo em Álgebra em Coimbra e quis dar a sua última lição, em 1987, sobre um tema de Matemática. Ocupou lugares de relevo na academia como os de Vice-Reitor de 1978 a 1982 e de Director da Biblioteca Geral da Universidade de Coimbra de 1978 até à data da sua jubilação. Foi um cidadão muito empenhado na cultura, tendo sido um dos protagonistas maiores na redacção da revista Vértice. Politicamente, para além da expressão das suas ideias de oposição e da grande abertura às dos outros, limitou-se a ser, por pouco tempo, governador civil de Coimbra após o 25 de Abril de 1974.

O seu liber amicorum, volume de homenagem para o qual contribuíram numerosos colegas e amigos, Luís de Albuquerque Historiador e matemático ${ }^{13}$, inclui, para além da sua extensa bibliografia, depoimentos de colegas e amigos ${ }^{14}$. Transcrevemos do depoimento do Professor de Matemática da Universidade de Lisboa J. J. Dionísio esta citação do Prof. Albuquerque sobre o ensino da História da Matemática, que ele ministrou em Coimbra:

"Para isso [ensinar aquela disciplina] é necessário que a uma sólida e ampla formação de matemático o professor alie um bom

\footnotetext{
9 Albuquerque, 1987.

10 Albuquerque, 1989.

11 Albuquerque, 1989.

12 Albuquerque, 1994.

13 Albuquerque, 1994.

${ }^{14}$ Marques,1998; Vários, 2007.
} 
conbecimento de história das ideias e das mentalidades, devendo ainda estar bem apetrechado na área das línguas."

O Prof. Albuquerque tentou até ensinar ao mesmo tempo alemão (ele tinha estado na Alemanha) e topologia: poder-se-á imaginar uma maneira mais original de juntar letras e ciências?

\section{Rómulo de Carvalho}

Por último, Rómulo de Carvalho, nascido em Lisboa, foi professor de Físico-Química, com formação adquirida primeiro na Universidade de Lisboa e depois na do Porto, onde concluiu os estudos, tendo-se revelado, para além de pedagogo e historiador da pedagogia em Portugal, um notável historiador e um incansável divulgador de ciência (ver os dois volumes saídos na Universidade de Évora ${ }^{15}$ ). Tal como Joaquim de Carvalho, Rómulo de Carvalho interessou-se pelo segundo período de ouro da ciência portuguesa: o Iluminismo, um tempo onde foi preponderante a acção dos sócios portugueses da Royal Society de Londres (muitos deles "estrangeirados" como o judeu Jacob de Castro Sarmento, mas também os oratorianos Teodoro de Almeida e João Chevalier e o originalmente crúzio João Jacinto Magalhães foram sócios dessa instituição ${ }^{16}$ ). Tendo a actividade científica desses "modernos" (assim se diziam os adeptos da física de Galileu e Newton) atingido um pico no final do reinado de D. João V (que, recorde-se, faleceu em 1750), ela sofreu um sobressalto com a perseguição primeiro aos jesuítas e depois aos oratorianos (quanto à perseguição aos judeus ela já vinha do tempo de D. Manuel I) após terramoto de Lisboa de 1755, mas

\footnotetext{
15 Carvalho, 1996; Carvalho, 1997.

16 Fiolhais, 2011.
} 
essa actividade acabou por encontrar acolhimento na Reforma da Universidade de Coimbra, em 1772, designadamente com a criação do Gabinete de Física, e, já depois da "Viradeira", com a inauguração da Academia de Ciências de Lisboa, em 1779. Vários professores de Ciências de Coimbra foram sócios fundadores dessa Academia, o que significa que, apesar do nome, o seu carácter nacional foi imediatamente reconhecido.

Para estudar esse período Rómulo foi um frequentador assíduo quer do antigo Gabinete de Física de Coimbra quer da Biblioteca Geral e do Arquivo da Universidade quando era professor no Liceu Normal de D. João III (hoje Escola Secundária José Falcão), em Coimbra. Mais tarde foi, durante muitos anos, frequentador da Biblioteca e Museu da Academia de Ciências, quando era professor no Liceu Pedro Nunes, em Lisboa. Como, na época, os estudos sobre os Descobrimentos já estavam em grande parte lançados, preferiu focar a sua atenção sobre esse outro grande período da ciência em Portugal que foi a recepção plena do newtonianismo, que é como quem diz, da ciência moderna. É talvez o nosso maior historiador da Ciência Iluminista, que divulgou nos pequenos volumes da colecção Biblioteca Breve do Instituto de Cultura e História Portuguesa ${ }^{17}$, hoje todos em acesso livre on line ${ }^{18}$ ) sobre a Astronomia, Física Experimental e História Natural no século XVIII.

Apesar de bem acolhido no Laboratório de Física pelo seu Director João de Almeida Santos, Rómulo de Carvalho não foi muito feliz nos contactos que manteve com a Universidade com vista à publicação de uma sua obra sobre a história do estabelecimento da Física Experimental com a fundação do Gabinete de Física pelo Marquês de Pombal, equipado em boa parte com máquinas vindas da rede

\footnotetext{
17 Carvalho, 1982; Carvalho, 1985; Carvalho, 1987.

18 Biblioteca Virtual Camões http://cvc.instituto-camoes.pt/ (acesso em 8 de Setembro de 2014)
} 
dos oratorianos de Lisboa ${ }^{19}$. Nas suas Memórias $^{20}$, publicadas pela Fundação Gulbenkian, conta como foram atribuladas as suas relações com José Sebastião da Silva Dias, Professor da Faculdade de Letras da Universidade de Coimbra (licenciado em Direito, Silva Dias começou em 1957 a dar aulas de História da Cultura nessa Faculdade, sucedendo de certo modo a Joaquim de Carvalho): o grosso volume sobre o Gabinete de Física pombalino (esse Gabinete é, desde 2014, "Sítio Histórico da Física" na Europa, por designação da Sociedade Europeia de Física ${ }^{21}$ ) acabou por ver a sua publicação protelada para sair finalmente do prelo pela Biblioteca Geral, no tempo em que era director desta Luís de Albuquerque, mas apenas quinze anos após a sua redacção e uma paragem de quase quatro anos na tipografia. A Universidade de Évora, ao conceder a Rómulo de Carvalho o grau de doutor honoris causa em 1995, prestou-lhe uma homenagem que, vinda do mundo universitário, foi tão justa quanto tardia. Em Coimbra, o nome do Rómulo - Centro Ciência Viva da Universidade de Coimbra ${ }^{22}$, moderno centro de recursos em cultura científica integrado no Instituto de Investigação Interdisciplinar da Universidade de Coimbra, também lhe presta homenagem. Como curiosidade, acrescente-se que Rómulo de Carvalho veio a ocupar em 1992 o lugar de Luís de Albuquerque na Academia das Ciências, tendo-lhe feito na altura o devido elogio.

Rómulo de Carvalho foi, como é bem sabido, o poeta António Gedeão. Haveria para ele uma dicotomia entre ciência e poesia? Respondeu esse homem das "duas culturas" 23 numa entrevista a

19 Carvalho, 1987; Fiolhais, 2008: 35-42

20 Carvalho, 2010.

${ }^{21} \mathrm{http} / / /$ www.uc.pt/colaboradores/destaques/20140901 (acesso em 8 de Setembro de 2014)

22 http://nautilus.fis.uc.pt/rc/?page_id=87 (acesso em 8 de Setembro de 2014)

23 Snow, 1996. 
Christopher Auretta e António Nunes dos Santos, publicada na Gazeta de Física ${ }^{24}$ :

$R C / A G-(\ldots)$ Há alguma dicotomia? Não há nenhuma! A pessoa encara a poesia como encara a ciência como encara a arte, como encara qualquer outra coisa, não há incompatibilidade.

CA - Convivem pacificamente?

$R C / A G$ - Com certeza.

CA - Enriquecem-se?

RC/AG - Exactamente. Não há nenhum motivo para me dizerem: "Então, você que é cientista, também faz poesia? Não tem nada uma coisa com a outra. Pois é, faço e também faço móveis!"

Referências sobre a vida e a obra de Rómulo de Carvalho / António Gedeão foram as exposições que o Museu de Ciência da Universidade de Lisboa e a Biblioteca Nacional de Portugal lhe fizeram postumamente, ambas com bons catálogos ${ }^{25}$.

\section{CONCLUSÕES}

O que têm em comum os Professores Joaquim de Carvalho, Luís de Albuquerque e Rómulo de Carvalho? Além de uma imensa capacidade intelectual, uma omnívora voragem pelos conhecimentos históricos, que ia sendo satisfeita pela consulta e leitura dos manuscritos e cimélios (todos foram distintos bibliófilos). E, sobretudo, todos eles prezaram o cruzamento entre as várias áreas do vasto saber humano.

${ }^{24} \mathrm{http}: / /$ nautilus.fis.uc.pt/spf/velharia/gazeta/93/GF-16_1.93/02.html (acesso em 8 de Setembro de 2014)

25 Rêgo, Manuela, et al (org.), (2006), sítio “António é o meu nome”, Lisboa: Biblioteca Nacional de Portugal http://purl.pt/12157/1/ (acesso em 8 de Setembro de 2014) e Corte-Real e Lourenço, 2001. 
Joaquim de Carvalho situa-se entre a filosofia e a ciência (o ramo da filosofia que dá pelo nome de epistemologia não é mais do que o enquadramento da ciência na filosofia), Luís de Albuquerque entre a Matemática e a História (a navegação astronómica, que desempenhou um papel essencial na descoberta do mundo nos séculos XV e XVI, não é mais do que um ramo da Matemática Aplicada) e Rómulo de Carvalho entre a ciência, a história, a pedagogia, a divulgação e a poesia (abrindo-se num espectro ainda maior do que os outros seus dois contemporâneos). Todos eles contribuíram para a História da Ciência na Universidade de Coimbra, que é em grande parte a História da Ciência em Portugal (ver os volumes recentes da Imprensa da Universidade de Coimbra $^{26}$ e a obra de divulgação histórico-científica ${ }^{27}$, que, de certo modo, oferecem resposta ao repto do prefácio às Actas do III Congresso Internacional de História das Ciências, transcrito no início). Todos eles pugnaram pela difusão da ciência sob a forma privilegiada do livro, Joaquim de Carvalho não só como autor mas também como editor da Imprensa da Universidade, e Luís de Albuquerque e Rómulo de Carvalho como autores de extensíssimas bibliografias.

E há mais em comum: os três foram excepcionais na defesa da ciência no século XX português, que, apesar de ter beneficiado dos enormes avanços nas ciências e tecnologias feitos no mundo, só a partir de 1974 viu reconhecido o papel da ciência e da tecnologia no desenvolvimento nacional. E os três sempre pugnaram pela unidade das ciências. Podem as ciências ser múltiplas e díspares, mas das suas ligações e do reconhecimento da sua unidade (veja-se, como referência para a descrição dessa unidade, a obra de Olga Pombo, professora de Filosofia das Ciências na Universidade de Lisboa ${ }^{28}$ ) resulta sempre compreensão acrescida. Num tempo de enorme fragmentação,

\footnotetext{
${ }^{26}$ Fiolhais, Martins e Simões, 2013a. Fiolhais, Martins e Simões, 2013b.

27 Fiolhais, 2013.

28 Pombo, 2006.
} 
podendo mesmo dizer-se pulverização, disciplinar, a unidade das ciências pode trazer esclarecimento adicional. A visão histórica ajuda: Se os Descobrimentos foram o prelúdio da Revolução Científica e o Iluminismo, época em que triunfou a ciência de Galileu e Newton, foi marcado pela publicação da Enciclopédia de d'Alembert e Diderot e pelo diálogo interdisciplinar nas academias então formadas, o tempo de hoje, com o cultivo da História das Ciências, pode beneficiar sobremaneira com a revisitação intelectual desse passado.

O Instituto de Investigação Interdisciplinar da Universidade de Coimbra, que reúne todos os centros de Investigação da Universidade de Coimbra, pode ser o lugar onde esse cultivo se concretize ou pelo menos o lugar que fomente esse cultivo, sendo decerto um bom auspício a recente criação, numa iniciativa conjunta das Universidades de Coimbra e Aveiro, de um programa doutoral em "História das Ciências e Educação Científica". Que as figuras de Joaquim de Carvalho, Luís de Albuquerque e Rómulo de Carvalho sejam a este respeito inspiradoras.

\section{REFERÊNCIAS}

Albuquerque, L. (1985), Os Descobrimentos Portugueses, Alfa e Selecções do Reader's Digest, Lisboa.

Albuquerque, L. (1987), Navegadores, Viajantes e Aventureiros Portugueses. Sécs. $X V$-XVI, Lisboa: Círculo de Leitores e Caminho, 1987, 2 vols.

Albuquerque, L. (dir) e Domingues, F. C. (coord.) (1994), Dicionário de História dos Descobrimentos Portugueses, Círculo de Leitores e Caminho, 2 vols, Lisboa.

Albuquerque, L. (dir.) (1989a), Biblioteca da Expansão Portugueses, Alfa, 50 vols, Lisboa.

Albuquerque, L. (dir.) (1989b), Portugal no Mundo, Alfa, 6 vols, Lisboa.

Albuquerque, L., Magalhães, A. M. e Alçada, I. (1991-1992), Os Descobrimentos Portugueses, 2 vols., Caminho, Lisboa.

Carvalho, J. (1987), Obra Completa, Vol. V, História e Crítica Literárias e História da Ciência, Fundação Calouste Gulbenkian, Lisboa.

Carvalho, R. (1982), A Física Experimental em Portugal no Século XVIII, Instituto de Cultura e História Portuguesa, Lisboa. 
Carvalho, R. (1985), A Astronomia em Portugal no Século XVIII, Instituto de Cultura e História Portuguesa, Lisboa.

Carvalho, R. (1986), Actividades científicas em Portugal no século XVIII, Universidade de Évora, Évora.

Carvalho, R. (1987a), A História Natural em Portugal no Século XVIII, Instituto de Cultura e História Portuguesa, Lisboa.

Carvalho, R. (1987b), História do Gabinete de Física pombalino da Universidade de Coimbra: desde a sua fundação (1772) até ao jubileu do professor italiano Giovanni Antonio Dalla Bella (1790), Universidade de Coimbra, Coimbra.

Carvalho, R. (1997), Colectânea de estudos históricos (1953-1994): cultura e actividades científicas em Portugal, Universidade de Évora, Évora.

Carvalho, R. (2010), Memórias, Fundação Calouste Gulbenkian, Lisboa.

Corte-Real, L. e Lourenço, Marta (dir.) (2001), Pedra Filosofal. Rómulo de Carvalho / António Gedeão, Museu de Ciência da Universidade de Lisboa, Lisboa.

Fiolhais, C. (2008), "Os Livros que Rómulo de Carvalho nos deixou", in Célia Vieira e Isabel Rio Novo (orgs.), Actas do Encontrro Internacional António Gedeão \& Rómulo de Carvalho, Novos Poemas para o Homem Novo, Edições ISMAI, Maia, 35-42.

Fiolhais, C. (2011) (coord.), Sócios Portugueses da Royal Society / Portuguese Fellows of the Royal Society, Universidade de Coimbra, Coimbra.

Fiolhais, C. (2013), História da Ciência em Portugal, Arranha Céus, Lisboa

Fiolhais, C., Martins, D. e Simões, C. (coord.) (2013a), História da Ciência Luso-Brasileira: Coimbra entre Portugal e o Brasil, Imprensa da Universidade, Coimbra.

Fiolhais, C., Martins, D. e Simões, C. (coord.) (2013b), História da Ciência na Universidade de Coimbra (1772-1933), Coimbra: Imprensa da Universidade, 2013b.

III Congrès (1936) como International d'Histoire des Sciences. Actes, Conferences et Communications, Lisboa.

Marques, A. P. (1998), Luís de Albuquerque na historiografia portuguesa: a serenidade e a convicção. Centro de Estudos do Mar, Coimbra e Figueira da Foz.

Nunes, P. (1940-1960), Obras / Pedro Nunes. - Nova edição, revista e anotada por uma Comissão de Sócios da Academia das Ciências, Imprensa Nacional, 4 vols, Lisboa.

Pombo, O. (2006), Unidade da Ciência. Programas, Figuras e Metáforas, Duarte Reis, Lisboa.

Sarton, G. (1931), The history of science and the new humanism, Henry Holt and Co., New York.

Rêgo, Manuela, et al (org.), (2006), "António é o meu nome", Rómulo de Carvalbo, Biblioteca Nacional, Lisboa.

Drey, J., et al, (2007), Testemunhos. Luís de Albuquerque, Ordem dos Engenheiros - Região Centro, Coimbra.

Contente, Domingos Francisco, (1998), Luís de Albuquerque. Historiador e Matemático, Homenagem, de Amizade a um Homem de Ciência, Chaves Ferreira - Publicações, Lisboa. 


\section{B I B L I O G RA FIA}

Adams, J. (2013), "The fourth age of research", Nature, 497: 557-560.

Albuquerque, L. (1985), "Os Descobrimentos Portugueses", Alfa e Selecções do Reader's Digest, Lisboa.

Albuquerque, L. (1987), "Navegadores, Viajantes e Aventureiros Portugueses", Sécs. XV-XVI, Lisboa: Círculo de Leitores e Caminho, 1987, 2 vols.

Albuquerque, L. (dir) e Domingues, F. C. (coord.) (1994), "Dicionário de História dos Descobrimentos Portugueses", Círculo de Leitores e Caminho, 2 vols, Lisboa.

Albuquerque, L. (dir.) (1989a), "Biblioteca da Expansão Portugueses", Alfa, 50 vols, Lisboa.

Albuquerque, L. (dir.) (1989b), "Portugal no Mundo", Alfa, 6 vols, Lisboa.

Albuquerque, L., Magalhães, A. M. e Alçada, I. (1991-1992), "Os Descobrimentos Portugueses", 2 vols., Caminho, Lisboa.

Altbach, P.G. (2008), "The complex roles of universities in the period of globalization", Higher Education in the World: New Challenges and Emerging Roles for Human and Social Development, P. Altbach, Basingstoke, UK, GUNI/Macmillan Palgrave, 2008.

Anderberg, M.R. (1973), "Cluster analysis for applications", Academic Press, New York.

Ardebili, M. (2005), "Reorienting Economics, by Tony Lawson - Book Review", Review of Social Economy, 651-657.

Arena, R.; Dow, S.; Klaes, M. (2009), "Open Economics: Economics in relation to other disciplines". Londres e Nova Iorque: Routledge.

Arthur, B. (2013), "Complexity Economics: A Different Framework for Economic Thought", Institute for New Economic Thinking (INET) Research Notes \#033 (disponível em http://ineteconomics.org/sites/inet.civicactions.net/files/Note33-Arthur.pdf)

Ash, A., Stephen, G. (1997), "The Ordinary City", Transactions of the Institute of British Geographers, 22, 4, 411-29.

Beauregard, R. (2003), "Voices of Decline: The postwar fate of US cities", Nova Iorque: Routledge.

Becker, G. (1976), “The Economic Approach to Human Behavior”, Chicago: University of Chicago Press.

Becker, G. (1993), "Gary S. Becker - Prize Lecture: The Economic Way of Looking at Life". (disponível em http://www.nobelprize.org/nobel_prizes/economicsciences/laureates/1992/becker-lecture.html)

Bernal, J. D. (1967), "Social Function of Science", Cambridge, Mass., MIT Press.

Bishop, R., Phillips, J., Wei Yeo, W. (2003), "Postcolonial urbanism: Southeastern Asian cities and global processes", Londres e Nova Iorque, Routledge.

Blackwell, A., Wilson, L., Street, A., Boulton, C., \& Knell, J. (2009), "Radical innovation: crossing knowledge boundaries with interdisciplinary teams", University of Cambridge, Technical Report, No. 760, UCAM-CL-TR-760, ISSN 1476-2986, 2009. 
Borges, A. (2010), "Religião e Diálogo Inter-Religioso", Imprensa da Universidade de Coimbra, Coimbra.

Carvalho, J. (1987), Obra Completa, Vol. V, "História e Crítica Literárias e História da Ciência”, Fundação Calouste Gulbenkian, Lisboa.

Carvalho, R. (1982), "A Física Experimental em Portugal no Século XVIII", Instituto de Cultura e História Portuguesa, Lisboa.

Carvalho, R. (1985), "A Astronomia em Portugal no Século XVIII", Instituto de Cultura e História Portuguesa, Lisboa.

Carvalho, R. (1986), "Actividades científicas em Portugal no século XVIII", Universidade de Évora, Évora.

Carvalho, R. (1987a), "A História Natural em Portugal no Século XVIII", Instituto de Cultura e História Portuguesa, Lisboa.

Carvalho, R. (1987b), "História do Gabinete de Física pombalino da Universidade de Coimbra: desde a sua fundação (1772) até ao jubileu do professor italiano Giovanni Antonio Dalla Bella (1790)", Universidade de Coimbra, Coimbra.

Carvalho, R. (1997), "Colectânea de estudos históricos (1953-1994): cultura e actividades científicas em Portugal”, Universidade de Évora, Évora.

Carvalho, R. (2010), "Memórias”, Fundação Calouste Gulbenkian, Lisboa.

Castells, M. (1972), "La Question Urbaine”, Paris: Maspero.

Chen et al. (2014), "Interdisciplinarity Patterns of Highly-Cited Papers: A CrossDisciplinary Analysis", S. Chen, C. Arsenault, Y. Gingras e V. Larivière, 77th ASIS\&T Annual Meeting, Seattle, WA, USA, October 31- November 4, 2014.

Chick, V.; Dow, S. (2005), "The meaning of open systems", Journal of Economic Methodology, 12, 3: 363-381.

Choi, B.C.K. e Pak, A.W.P. (2006), "Multidisciplinarity, interdisciplinarity and transdisciplinarity in health research, services, education and policy: Definitions, objectives, and evidence of effectiveness", Clinical \& Investigative Medicine journal, Vol. 29, no 6, December 2006.

Coase, R. (1994 [1977]), "Economics and Contiguous Disciplines", in Coase, Ronald, Essays on Economics and Economists. Chicago: The University of Chicago Press, $34-46$.

Corte-Real, L. e Lourenço, Marta (dir.) (2001), "Pedra Filosofal, Rómulo de Carvalho /António Gedeão", Museu de Ciência da Universidade de Lisboa, Lisboa.

Couto-Soares, M. L. (2013), "A estrutura do conhecimento tácito em Polanyi; um paradigma pós-crítico para a epistemologia?”, in http://mlag.up.pt/wp-content/ uploads/2012/01/Conhecimento-Tácito.pdf; acesso em 14 de Dezembro de 2013.

Cova, T. F. G. G., Pereira, J. L. G. F. S. C. e Pais, A. A. C. C., "Is standard multivariate analysis sufficient in clinical and epidemiological studies?" Journal of Biomedical Informatics, 46: 75-86.

Davies, M. C. e Fearn, T. (2004), "Back to basics: the principles of Principal Component Analysis”, Spectroscopy Europe, 16: 20-23.

Davis, J. (2006), "The turn in economics: neoclassical dominance to mainstream pluralism?", Journal of Institutional Economics, 2, 1: 1-20. 
Dequech, D. (2012), "Economia e Sociologia Econômica: Abordagens, Objetos e Práticas", in Kerstenetzky, Celia; Neves, Vítor (orgs.), Economia e interdisciplinaridade(s). Coimbra: Edições CES/Almedina, 149-172.

DGEEC (2014), "Produção Científica Portuguesa, 1990-2013: Séries Estatísticas", Direção-Geral de Estatísticas de Educação e Ciência (DGEEC), 2014, disponíveis online em http://www.dgeec.mec.pt/np4/457.html.

Diamond, P.; Vartiainen, Hannu (eds.) (2007), "Behavioral Economics and its Applications". Princeton e Oxford: Princeton University Press.

Downs, A. (1957), "An economic theory of democracy", Nova Iorque: Harper and Row.

Escobar, A. (1995), "Encountering development: The making and unmaking of the third world", Princeton: Princeton University Press.

EURAB (2004), "Interdisciplinarity in Research", report from the European Union Research Advisory Board, EURAB, 2004 (disponível em: http://ec.europa.eu/ research/eurab/pdf/eurab_04_009_interdisciplinarity_research_final.pdf).

Fine, B. (1997), "The new revolution in Economics", Capital \& Class 61: 143-148.

Fine, B. (2002), “Economic Imperialism': a view from the periphery”, Review of Radical Political Economics, 34: 187-201.

Fiolhais, C. (2008), "Os Livros que Rómulo de Carvalho nos deixou”, in Célia Vieira e Isabel Rio Novo (orgs.), Actas do Encontrro Internacional António Gedeão \& Rómulo de Carvalho, Novos Poemas para o Homem Novo, Edições ISMAI, Maia, 35-42.

Fiolhais, C. (2011), "Sócios Portugueses da Royal Society", Universidade de Coimbra, Coimbra.

Fiolhais, C. (2013), "História da Ciência em Portugal", Arranha Céus, Lisboa

Fiolhais, C., Martins, D. e Simões, C. (coord.) (2013a), "História da Ciência Luso-Brasileira: Coimbra entre Portugal e o Brasil", Imprensa da Universidade, Coimbra.

Fiolhais, C., Martins, D. e Simões, C. (coord.) (2013b), "História da Ciência na Universidade de Coimbra (1772-1933)", Coimbra: Imprensa da Universidade, 2013b.

Fontana, M. (2009), "The Santa Fe Perspective on Economics: emerging patterns in the science of complexity", WP CESMEP Working paper No. 08/2009 (disponível em http://www.cesmep.unito.it/WP/2009/8_WP_Cesmep.pdf)

Formosinho, S. J., "Uma Intuição por Portugal”, Artez.

Fortuna, C. (1997), "As cidades e as identidades: Narrativas, patrimónios e memória", Revista Brasileira de Ciências Sociais, 33, 127-141.

Fortuna, C. (2012a), "In praise of other views: The world of cities and the social sciences". Iberoamericana, XII, 45, 137-153.

Fortuna, C. (2012b), Apresentação, In Lefebvre, Henri, "O direito à cidade". Lisboa, Letra Livre/Estúdio.

Frey, B. e Benz, M. (2004), "From imperialism to inspiration: a survey of Economics and psychology", in Davis, John et al. (eds.), The Elgar Companion to Economics and Philosophy. Cheltenham: Edward Elgar, 61-83. 
Friedman, J. (1986), "The World City Hypothesis", Development and Change, 17, 69-83.

Gonçalves-Maia, R. (2013), "Dos Raios X à Estrutura Biomolecular", Editora Livraria de Física, São Paulo, Brasil.

Graça, J. C. e Lopes, . C. (2010), "Complexidade, interdependência e autonomia em sistemas económicos e sociais", Revista Crítica de Ciências Sociais [Online], 90 | 2010 (disponível em http://rccs.revues.org/1777)

Groenewegen, P. (2007), "Alfred Marshall: Economist 1842-1924”, Hampshire, UK e Nova York: Palgrave Macmillan. [tradução portuguesa: Alfred Marshall, Lisboa: Actual Editora, 2011]

H2020 (2013) "Establishing Horizon 2020 - the Framework Programme for Research and Innovation (2014-2020)", Official Journal of the European Union, L 347/104, 20/12/2013.

Hannerz, U. (1980), "Exploring the City. Inquiries Toward an urban anthropology", Nova Iorque, Columbia University Press.

Harvey, D. (1989), "The condition of postmodernity", Oxford, Blackwell.

Hastie, T., Tibshirani, R. e Friedman, J. (2001), "The elements of statistical learning: data mining, inference and prediction", Springer, 2001.

Hirshleifer, J. (1985), “The Expanding Domain of Economics”, The American Economic Review, 75, 6: 53-68.

Hodgson, G. (1996), "Towards a Worthwhile Economics", in Medema, Steven; Samuels, Warren (eds.), How Economists Should Do Economics. Aldershot: Edward Elgar, 103-21.

Hodgson, G. (2001), "How economics forgot history: the problem of historical specificity in social science", Londres e Nova Iorque: Routledge.

Hodgson, G. (2008), "Marshall, Schumpeter and the shifting boundaries of economics and sociology", in Shionoya, Yuichi; Nishizawa, Tamotsu (eds.), Marshall and Schumpeter on Evolution: Economic Sociology of Capitalist Development, Cheltenham, UK; Northampton, MA, USA: Edward Elgar.

Hollis, L. (2013), "Cities are good for you: The genius of the metropolis", Londres, Nova Deli, Nova Iorque e Sydney, Bloomsbury.

Holt, R., Rosser Jr., B., Colander, D. (2011), "The Complexity Era in Economics", Review of Political Economy, 23, 3: 357-369.

III Congrès (1936) como International d'Histoire des Sciences. Actes, Conferences et Communications, Lisboa.

Ingham, G. (1996), "Some recent changes in the relationship between economics and sociology", Cambridge Journal of Economics, 20: 243-275.

Joliffe, I. T. (2 $2^{a}$ ed. 2002), "Principal Component Analysis", Springer, New York.

Kapp, K. W. (1961), "Toward a Science of Man in Society: A Positive Approach to the Integration of Social Knowledge", The Hague: Martinus Nijhoff.

Kapp, K. W. (1976), "The Open-System Character of the Economy and its Implications", in Dopfer, Kurt (org.), Economics in the Future. Londres: The Macmillan Press, 90-105. 
Kapp, K. W. (1985), "The Humanization of the Social Sciences", organizado por Ullmann, John; Preiswerk, Roy, Lanham: University Press of America.

Kaufman, L., Rousseeuw, P. J. (1990), "Finding Groups in Data: An Introduction to Cluster Analysis”, Wiley, New York, 1990.

Kerstenetzky, C. (2012), "Economia e Ética: a virada normativa?", in Kerstenetzky, Celia; Neves, Vítor (orgs.), Economia e interdisciplinaridade(s). Coimbra: Edições CES/Almedina, 55-76.

Kerstenetzky, C.; Neves, V. (orgs.) (2012), "Economia e Interdisciplinaridade(s)", Coimbra: Almedina.

Larivière, V. (2015) "Long-Distance Interdisciplinarity Leads to Higher Scientific Impact", Vincent Larivière, Stefanie Haustein e Katy Börner, Open Access journal, PLOS ONE, published online on Mar 30th, 2015.

Larivière, V. e Gingras, Y. (2014), "Measuring inter-disciplinarity", in B. Cronin \& C. Sugimoto (Eds.), Beyond Bibliometrics: Harnessing Multidimensional Indicators of Scholarly Impact (pp. 187-200). Cambridge, Mass.: MIT Press, 2014.

Larivière, V. e Rafols, I. (2011), "Indicators of the Interdisciplinarity of Journals: Diversity, Centrality, and Citations", Journal of Informetrics, Volume 5, Issue 1, Pages 87-100, January 2011.

Lawson, T. (2003), \#Reorienting economics", Londres e Nova Iorque: Routledge.

Lazear, E. (2000), "Economic Imperialism", The Quarterly Journal of Economics, 115, $1,99-146$

Lefebvre, H. (2012) [1968], "O direito à cidade”, Lisboa, Letra Livre/Estúdio.

Loasby, B. (1999), "Knowledge, Institutions and Evolution in Economics", Londres e Nova Iorque: Routledge.

Loasby, B. (2003), "Closed models and open systems", Journal of Economic Methodology, 10, 3: 285-306.

Mäki, U. (1992), "On the Method of Isolation in Economics", Poznan Studies in the Philosophy of the Sciences and the Humanities, 26, 317-351.

Mäki, U. (2001), "The way the world works (www): towards an ontology of theory choice”, in Mäki, Uskali (ed.), The Economic World View: Studies in the Ontology of Economics, Cambridge: Cambridge University Press, 369-389.

Mäki, U. (2004), "Theoretical isolation and explanatory progress: transaction cost economics and the dynamics of dispute", Cambridge Journal of Economics, 28, 319-346.

Marques, A. P. (1998), "Luís de Albuquerque na historiografia portuguesa: a serenidade e a convicção". Centro de Estudos do Mar, Coimbra e Figueira da Foz.

Martins, J. P. O. (13 $3^{a}$ ed. 2007), "História da Civilização Ibérica, edição compulsada", Guimarães Editores Lda.

Mendieta, E. (2001), "Invisible Cities: A Phenomenology of Globalization From Below", City, 5 , 1, 7-26.

Mirowski, P. (1991), "The When, the How and the Why of Mathematical Expression in the History of Economics Analysis", The Journal of Economic Perspectives, 5, 1: 145-157. 
Moessner, L. (2009), "The influence of the Royal Society on 17 th-century scientific writing”, ICAME Journal, 33: 65-87, 2009.

Mullins, P. (2004), "Bible Study, Critical Thinking and Post-Critical Thought: Cultural Considerations", in Charles M. Ess (ed.), Critical Thinking and the Bible in the Age of the New Media,. Lanham, MD: University Press of America: 269-290.

Neves, V. (2012a), "A análise dos custos sociais em Ronald Coase e K. William Kapp: duas perspectivas sobre a economia e a interdisciplinaridade", in Kerstenetzky, Celia; Neves, Vítor (orgs.), Economia e interdisciplinaridade(s). Coimbra: Edições CES/Almedina, 125-147.

Neves, V. (2012b), "Sciences as Open Systems - The Case of Economics", in Pombo et al. (eds.), Special Sciences and the Unity of Science. Dordrecht: Springer, 205-217.

Nordvig, J. (2014), "A Queda do Euro", Casa das Letras, Alfragide.

Nunes, A. S. (1976 4ª ed.), "Questões Preliminares sobre as Ciências Sociais", Lisboa: Gabinete de Investigações Sociais.

Nunes, P. (1940-1960), Obras / Pedro Nunes. - Nova edição, revista e anotada por uma Comissão de Sócios da Academia das Ciências, Imprensa Nacional, 4 vols, Lisboa.

Ortega y Gasset, J. (1930), “Misión de la Universidad”, (disponível em: http://www.esi2. us.es/ fabio/mision.pdf).

Peixoto, P. (1989), "Alguns dados sobre o ensino superior em Portugal", Revista Crítica de Ciências Sociais, $n^{\circ}$ 27/28, junho de 1989.

Polanyi, M. (1973), "Personal Knowledge. Towards a Post-Critical Philosophy", Routledge \& Kegan Paul, London, 1958, ed. compulsada paperback 1973.

Pombo, O. (2004a), "Interdisciplinaridade: Ambições e Limites", Lisboa: Relógio d'Água.

Pombo, O. (2004b), "Epistemologia da interdisciplinaridade", in Pimenta, Carlos (Org.), Interdisciplinaridade, Humanismo, Universidade. Porto: Campo das Letras, 93-124.

Pombo, O. (2006), "Unidade da Ciência. Programas, Figuras e Metáforas", Duarte Reis, Lisboa.

Porter, A. e Rafols, I (2009), "Is science becoming more interdisciplinary? Measuring and mapping six research fields over time", Scientometrics, Vol. 81, No. 3 pp. 719-745, 2009.

Posner, R. (1972 1st edition), "Economic Analysis of Law", Boston: Little, Brown and Company.

Potts, J. (2000), "The New Evolutionary Microeconomics: Complexity, Competence and Adaptive Behaviour", Cheltenham: Edward Elgar.

Prager, K., Morris, S., Currie, M., Macleod, K. (2015), "Exploring Interdisciplinarity: summary report of the "Developing an interdisciplinary culture of excellence (DICE)' project at the James Hutton Institute", The James Hutton Institute, Scotland, UK, 2015.

Radhakrishna Rao, C. (1982), "Diversity: Its measurement, decomposition, apportionment and analysis", Sankhy : The Indian Journal of Statistics, Series A, 44(1), 1-22, 1982. 
Rafols, I., Leydesdorff, L., O'Hare, A., Nightingale, P., Stirling, A. (2012), "How journal rankings can suppress interdisciplinary research: A comparison between Innovation Studies and Business \& Management", Research Policy journal, 41(7), 2012.

Rafols, I., Meyer, M. (2010), "Diversity and network coherence as indicators of interdisciplinarity: case studies in bionanoscience", Scientometrics, February 2010, Volume 82, Issue 2, pp 263-287.

Roberts, B. (1978), "Cities of peasants. The political economy of urbanization in the third world", Beverly Hills e Londres, Sage.

Robinson, J. (2002), "Global and world cities: A view from off the map", International Journal of Urban and Regional Research, 26, 3, 531-54.

Robinson, J. (2006), “Ordinary cities: Between modernity and development”, Londres e Nova Iorque, Routledge.

Rollo, M. F., Meireles, P., Ribeiro, M., Brandão, T. (2012), "História e Memória da Ciência e da Tecnologia em Portugal. O Arquivo de Ciência e Tecnologia da Fundação para a Ciência e a Tecnologia”, Boletim do Arquivo da Universidade de Coimbra, XXV [2012], pp. 233-261, 2012.

Santos, B. S. (1986-1986), "Discursos. Abertura Solene das Aulas da Universidade de Coimbra, 4 de Dezembro de 1985", Anuário da Universidade de Coimbra.

Santos, A. (2010), "Terá chegado o tempo dos arquitectos e designers económicos?", in Neves, Vítor; Caldas, José, A Economia Sem Muros. Coimbra: Edições CES/ Almedina, 117-138.

Santos, A. (2012), "Entre a Economia e a Psicologia: comportamento e experiências", in Kerstenetzky, Celia; Neves, Vítor (orgs.), Economia e interdisciplinaridade(s). Coimbra: Edições CES/Almedina, 173-196.

Santos, B. S. (2014), "Epistemologies of the South: Justice against Epistemicide", Boulder (CO), Paradigm Publishers.

Sarton, G. (1931), "The history of science and the new humanism", Henry Holt and Co., , New York.

Sassen, S. (2001), "The Global City: New York, London, Tokyo", Princeton: Princeton University Press.

Sassen, S. (2007), "Reading the city in a global digital age: The limits of topographic representation", in Taylor, Peter; Ben Derudder; Pieter Saey and Frank Witlox (eds.), Cities in Globalization: Practices, policies and theories. Londres e Nova Iorque, Routledge, 239-257.

Sharon M., M. (2007), "Philosophy in the Streets: Walking the City with Engels and de Certeau", City, 11, 1, 7-20.

Sneath, P.H.A. e Sokal, R. R. (1973), "Numeric taxonomy: the principles and practice of numerical classification", San Francisco: W. H. Freeman, 1973.

Soja, E., Kanai, M. (2007), "The urbanization of the world”,In Burdett, Ricki e Sudjic, Deyan (orgs.), The endless city. Londres, Phaidon, 54-69.

Stanley, L. J. (1978), "The Road of Science and the Ways to God", Univ. of Chicago Press, and Edinburgh: Scottish Academic Press.

Stirling, A. (2007), "A general framework for analysing diversity in science, technology and society", Journal of the Royal Society Interface, 4(15), 707, 2007. 
Strober, M. (2006), "Habits of the Mind: Challenges for Multidisciplinary Engagement", Social Epistemology: A Journal of Knowledge, Culture and Policy, 20, 3-4, 315331.

Strober, M. (2011), "Interdisciplinary conversations: challenging habits of thought", Stanford, CA: Stanford University Press.

Sugden, R. (2001), "The evolutionary turn in game theory", Journal of Economic Methodology, 8, 1: 113-130.

Swedberg, R. (1990), "The New 'Battle of Methods'”, Challenge, 33, 1: 33-38.

Syed Farid, A. (2003), "Academic Dependency and Global Division of Labour in the Social Sciences", Current Sociology, 51, 6, 599-613.

Thomas L. Hankins (2004), "Ciência e Iluminismo", Porto Editora, ISBN: 9789720450852 , 2004.

United Nations, Department of Economic and Social Affairs (Population Division) (2013), "World Urbanization Prospects: The 2011 Revision", Nova Iorque, United Nations.

Vaggi, G. e Groenewegen, P. (2003), "A Concise History of Economic Thought From Mercantilism to Monetarism", Hampshire, UK e Nova York: Palgrave Macmillan.

van Damme, E. (1999), "Game Theory: the Next Stage," in Kirman, A.; Gérard-Varet, L-A. (eds.), Economics Beyond the Millennium. Oxford: Oxford University Press, 184-214.

Van den Besselaar, P. e L. Leydesdorff (1996), "Mapping Change in Scientific Specialties: A Scientometric Reconstruction of the Development of Artificial Intelligence", P. Van den Besselaar e L. Leydesdorff, Journal of the American Society for Information Science, 47, 415-436, 1996.

Rêgo, Manuela, et al (org.), (2006), "António é o meu nome", Rómulo de Carvalho, Bibllioteca Nacional, Lisboa.

Drey, J., et al, (2007), Testemunhos. Luís de Albuquerque, Ordem dos Engenheiros - Região Centro, Coimbra.

Contente, Domingos Francisco, (1998), "Luís de Albuquerque. Historiador e Matemático, Homenagem, de Amizade a um Homem de Ciência”, Chaves Ferreira - Publicações, Lisboa.

Velthuis, O. (1999), "The Changing Relationship Between Economic Sociology and Institutional Economics: From Talcot Parsons to Mark Granovetter", American Journal of Economics and Sociology, 58, 4: 629-649.

Wallerstein, I. (2001), "Unthinking Social Science: The limits of the nineteenth-century paradigms", Filadélfia, Temple University Press.

Zhenhua, Y. (2010), “'Being in the World' in a Polanyian Perspective”, in Knowing and Being. Perspectives on the Philosophy of Michael Polanyi, Tihamér Margitay (ed.), Cambridge Scholars Publishing: 50-67. 
OBRA PUBLICADA

COMA COORDENAÇÃO

CIENTÍFICA

$\mathrm{ECH}$

- U 